\title{
Gestational diabetes: a non-entity?
}

\author{
R J Jarrett
}

\begin{abstract}
Screening for gestational diabetes is commonly recommended despite the absence of a common definition of gestational diabetes. Furthermore, there is no consensus about management or treatment. Those who recommend screening do so largely on the basis of fetal morbidity, which seems to be predominantly "macrosomia"-another term without an agreed definition. The implications of macrosomia in terms of actual morbidity are also not clear. R J Jarrett reviews the history of the subject and concludes that gestational diabetes is simply impaired glucose tolerance temporally associated with pregnancy. Its main importance is as a predictor of subsequent non-insulin dependent diabetes, but it fails the major tests for a condition suitable for a screening programme.
\end{abstract}

In 1979 the National Diabetes Data Group produced a new system of classification for diabetes mellitus. ' In the following year a World Health Organisation expert committee generally approved this classification, ${ }^{2}$ with one important exception-gestational diabetes. The National Diabetes Data Group regarded gestational diabetes as a state of glucose intolerance first discovered during pregnancy. It was to be defined by criteria originally suggested by $\mathrm{O}$ 'Sullivan and Mahan ${ }^{3}$ and based on a three hour oral glucose tolerance test with a $100 \mathrm{~g}$ oral glucose load. (Actually the criteria had to be arbitrarily adjusted to take account of modern laboratory technology, which measures "true glucose" and uses plasma instead of whole blood.) The WHO expert committee recommended using the criteria derived for impaired glucose tolerance in the nonpregnant state and measured with a $75 \mathrm{~g}$ two hour oral glucose tolerance test.

This lack of consensus has continued into the 1990s. The WHO study group of 1985 reiterated the expert committee's recommendation, ${ }^{4}$ while the American Diabetes Association ${ }^{5}$ and the second international workshop conference on gestational diabetes ${ }^{6}$ supported the National Diabetes Data Group.

\section{History}

The reason(s) for this lack of consensus must be sought in the early history of the topic. After the end of the second world war screening for what is now called non-insulin dependent diabetes mellitus became increasingly popular, particularly in North America, and the first formal population study was carried out in Oxford, Massachusetts. These activities generated the problems of defining non-insulin dependent diabetes mellitus and the criteria for it, and until the 1980s many official and unofficial criteria were used.

The risk of non-insulin dependent diabetes in women was also thought to be related both to increased parity and to fetal stillbirth, as well as to increased incidence of heavy babies. ${ }^{7}$ Indeed, Gilbert and Dunlop suggested that there was a metabolic disturbance in the mother active for as long as 20 years before diabetes was diagnosed. ${ }^{8}$ It was presumably these data and the related hypotheses which led the United States Public Health Service unit in Boston, Massachusetts, to begin a series of studies of glucose tolerance and related factors in pregnant women, which led to the criteria for gestational diabetes of O'Sullivan and Mahan. ${ }^{3}$ These were derived from oral glucose tolerance tests in pregnant women attending the Boston City Hospital who were followed for up to six years with yearly oral glucose tolerance tests. Diabetes was defined using the United States Public Health Service criteria-much softer than current criteria for noninsulin dependent diabetes - and the criteria for gestational diabetes determined in relation to an arbitrary degree of prediction using the pregnancy glucose tolerance tests.

With hindsight, the authors were describing pregnancy associated impaired glucose tolerance, which, like gestational diabetes, is an unstable condition associated with age, obesity, and an increased risk of non-insulin dependent diabetes mellitus. ${ }^{89}$ Using data from the National Health and Nutrition Examination in the United States Harris has argued that the prevalence of impaired glucose tolerance or gestational diabetes in pregnant women is about that expected, when adjusted for age, from the prevalence observed in non-pregnant women. ${ }^{9}$ She reviewed the literature and concluded that if pregnancy had any effect on oral glucose tolerance it must be trivial.

\section{Implications for morbidity}

If then gestational diabetes is simply impaired glucose tolerance associated with pregnancy, does it have any implications for the index pregnancy? The women with gestational diabetes in the original Boston studies had higher perinatal mortality, though the difference was not statistically significant and the published analyses did not sufficiently examine the potential confounding variables, of which age and obesity were the most obvious. Subsequent data from Belfast during the years when overall perinatal mortality declined sharply suggest that any putative risk associated with gestational diabetes must be small, ${ }^{10}$ and it would require very large numbers of observations to provide statistical significance and to adjust for confounding variables.

In any case the protagonists of screening for and treatment of gestational diabetes do not use perinatal mortality among their arguments but point instead to greater morbidity. This seems to consist, predominantly, of a higher frequency of large for dates babies and of a high risk of caesarean section. Both are in part due to the association of gestational diabetes with obesity, though the caesarean section rate, which is high generally in the United States, is undoubtedly increased when the obstetrician is aware of the diagnosis. The association between blood glucose concentrations and fetal weight is lost when adjustment is made for maternal weight and age. ${ }^{1112}$
RJ Jarrett, emeritus profess of clinical epidemiology

BMF 1993;306:37-8 
Nevertheless, "macrosomia" and its prevention are a common concern among writers on gestational diabetes. Though frequently used, macrosomia has no consensual definition, the most usual being a fetal weight of $4000 \mathrm{~g}$ or more. However, after reviewing the obstetric literature Ales and Santini concluded that more than $4500 \mathrm{~g}$ would be a more appropriate criterion in relation to problems at delivery.

\section{Effects of treatment}

Can fetal weight be altered by treatment? O'Sullivan et al compared insulin or diet with usual care in one trial with random allocation ${ }^{13}$ and in a second with alternate allocation of treatment. ${ }^{14}$ In the second trial insulin or diet was associated with significantly fewer heavy babies, the cut off being $4100 \mathrm{~g}$. Coustan and Lewis compared insulin and diet, diet only, and usual care in what they claimed was a randomised study, ${ }^{15}$ though 20 of their 72 subjects were not randomly allocated. With macrosomia defined as a birth weight of more than $3860 \mathrm{~g}$, the incidence of macrosomia was significantly lower in the insulin treated patients, but there was no significant difference in mean birth weight. In none of these studies was the possible effect of confounding variables examined.

Whether or not insulin or diet reduces the incidence of heavy babies, authors clearly feel that the attempt should be made. However, the negative associations between birth weight and various chronic disorders of adult life shown by Barker and colleagues ${ }^{1617}$ provide new reasons for requiring that the gestational diabetes dogma should be justified by clear evidence of benefit.

\section{Screening}

Should we screen for gestational diabetes? Yes, and all pregnant women, according to the American Diabetes Association. ${ }^{5}$ Yes, but only for women aged over 30, according to the American College of Obstetricians and Gynaecologists. ${ }^{18}$ But do even Americans observe these recommendations? Two investigations of obstetricians and maternofetal specialists in the United States found notable differences in practice between and within the two groups of specialists in both screening and management of gestational diabetes. ${ }^{19} 20$

Screening is, of course, supposed to be subject to certain general rules. The two most important-that the disorder should be well defined and that it should be serious and there must be an effective way to treat or prevent it which could not be achieved without screening - are not satisfied for gestational diabetes.

Given the lack of an accepted definition and of reputable evidence about treatment other criteria for worthwhile screening programmes, such as prevalence, cost effectiveness, and screening test performance, cannot be evaluated.

\section{Conclusions}

Firstly, gestational diabetes is no more than a special case of impaired glucose tolerance, temporally associated with pregnancy.

Secondly, though this condition is associated with an increased statistical risk of subsequent non-insulin dependent diabetes, any maternofetal morbidity is more likely to be due to maternal age or obesity or, indeed, to the effects and consequences of diagnosis than to the glucose intolerance.

Finally, screening for gestational impaired glucose tolerance does not accord with any of the usual requirements for a worthwhile screening programme. Indeed, in engendering alarm in the screened population it probably does harm rather than good.

1 National Diabetes Data Group. Classification and diagnosis of diabetes mellitus and other categories of glucose intolerance. Diabetes 1979;28: 1038-57

2 WHO Expert Committee on Diabetes Mellitus. Second report. Geneva: WHO, 1980. (Technical Report Series 646.)

3 O'Sullivan JB, Mahan C. Criteria for the oral glucose tolerance test in pregnancy, Diabetes 1964:13:278-85.

4 WHO Study Group on Diabetes Mellitus. Repor. Geneva: WHO, 1985. (Technical Report Series 727.)

5 American Diabetes Association Inc. Position statement on gestational diabetes mellitus. Diabetes Care 1991;14:5-6.

6 Summary and recommendations of the second international workshopconference on gestational diabetes mellitus. Diabetes 1985;34:123-6.

7 Ales KL, Santini DL. Should all pregnant women be screened for gestational glucose intolerance? Lancet 1989;i:1187-91.

8 Gilbert JAL, Dunlop DM. Diabetic fertility, maternal mortality, and foetal loss rate. $B M \mathcal{F}^{1}$ 1949;i:48-51.

9 Harris MI. Gestational diabetes may represent discovery of pre-existing glucose intolerance. Diabetes Care 1988;11:402-11.

10 Hadden DR. Screening for abnormalities of carbohydrate metabolism in pregnancy 1966-1977; the Belfast experience. Diabetes Care 1980;3:440-6.

11 O'Sullivan JB, Gellis SS, Tenney BO. Gestational blood glucose levels in normal and potentially diabetic women related to the birth weight of their infants. Diabetes 1966;15:466-70.

12 Green JR, Schumacher LB, Pawson IG, Partridge JC, Kretchmer N. Influence of maternal body habitus and glucose tolerance on birth weight. Obstet Gymecol 1991;78:235-40.

13 O'Sullivan JB, Charles D, Dandrow RV. Treatment of verified pre-diabetics in pregnancy. F Reprod Med 1971;7:21-4

4 O'Sulivan JB. Prospective study of gestational diabetes and its treatment. In: Stowers JM, Sutherland HW, eds. Carbohydrate metabolism in pregnancy and the newborn. Edinburgh: Churchill Livingstone, 1975:195-204.

15 Coustan DR, Lewis SB. Insulin therapy for gestational diabetes. Obstet Gynecol 1978;51:306-10.

16 Barker DJP, Martyn CN. The maternal and fetal origins of cardiovascular disease. I Epidemiol Community Health 1992;46:8-11.

17 Hales CN, Barker DJP, Clark PMS, Cox LJ, Fall C, Winter PD. Fetal and infant growth and impaired glucose tolerance at age 64. BMf 1991;303: infant grow

18 American College of Obstetricians and Gynecologists. Technical bulletin. Management of Diabetes in Pregnancy 1986;92:1-5.

19 Furman GI, Steinberg MC. Diabetes screening during pregnancy. Diabetes 1987;36(suppl 1):90.

20 Gabbe SG, Landon MB. Management of diabetes mellitus in pregnancy: survey of materno-fetal subspecialists in the United States. In: Sutherland HW, Stowers JM, Pearson DWM, eds. Carbohydrate metabolism in pregnancy and the newborn. IV. London: Springer, 1989:309-17.

(Accepted 23 October 1992)

\section{ANY QUESTIONS}

Are some patients likely to be allergic to the clips used in surgery? Does it matter?

Clips are made either of stainless steel or rare metals such as tantalum or titanium. Though cutaneous reactions to such metals as steel, aluminium, and nickel (though not titanium) are well recognised, they are rare. Surprisingly, the implantation of metallic foreign bodies deep to the skin seems not to be associated with the same type of response. Any foreign body provokes some inflammatory reaction, though with the metals commonly used this is mild, is incorporated into the wound healing process, and does not seem to express itself in any general way as might be found in an allergic reaction. More subtle long term reactions may occur with large metallic prostheses and possibly relate to the ionic and electrochemical interaction of different metallic fragments, which may be shed from tools. This does not apply to clips.

The answer to the first question is, on the available evidence, no; and to the second, a slightly qualified no. But the increasing use of implanted clips should continue to be monitored, especially in certain sites such as bowel anastomoses, in which there is some tentative evidence that they may interact with residual malignant cells.-HUGH DUDLEY, emeritus professor of surgery, London 